\title{
Large Volume High Resolution FIB Nanotomography
}

\author{
Jason Huang
}

Carl Zeiss NTS LLC., one corporation way, Peabody MA 01960

3-dimensional (3D) imaging capability at the nanoscale has emerged as an important and powerful characterization technique in a wide variety of applications ranging from nanomaterials analysis in materials science to biological imaging in life science. Existing 3D imaging techniques, such as X-ray tomography, TEM tomography and Atom probe tomography all have their advantages and disadvantages when it comes to characterization at the nanoscale. While X-ray tomography can investigate relatively large (100um and more in lateral dimension) structures in a non-destructive way, it lacks the resolution to define nanoscale features; And both TEM and Atom probe tomographic techniques require intensive sample preparation, and the size limits imposed on the specimen exclude many interesting scientific topics. For example, a biological cell typically ranges from a few microns to tens of microns in diameter, which are beyond the penetration depth of a typical high energy beam in TEM $(80-300 \mathrm{kV})$.

Nanotomography based on serial FIB-SEM imaging has therefore become a viable imaging solution to fill in the gap between the resolution and the specimen sizes.

FIB nanotomography involves fully automated, concerted operations of the milling ion beam and the imaging e-beam. While this technique has been proposed for almost a decade [1], there are still challenges to fully utilize it as a mainstream tomography technique. For example, a medium size biological cell (see figures 1 and 2 for example), or a metallurgical structure, on the order of 40-60 um in diameter, could routinely demand days of unattended serial milling and imaging to scan through the volume of interest. Drifting in specimen position, focus and stigmation could be potential issues for such long runs. Large field of view as they may require, the smallest features of interest could be as small as a few nanometers, such as the lipid-bilayer in the cell membrane or a gas-permeable nanopore in a shale rock.

In this paper, an advanced FIB nanotomography platform will be presented. Application examples on biological science and material science will be shown to demonstrate: 1) fully automated FIB-SEM operations including automatic drift correction, ion beam regeneration, auto-focus and auto-stigmation for the imaging beam; 2) large store resolution (up to $32 \mathrm{k} x 32 \mathrm{k}$ ) for high resolution imaging. Such functionalities are tailored to handle large volume, high resolution imaging needs in both materials science and life science.

Reference:

[1] M. Phaneuf and J. Li, Microscopy and Microanalysis, Vol. 6, 524-525, 2000 


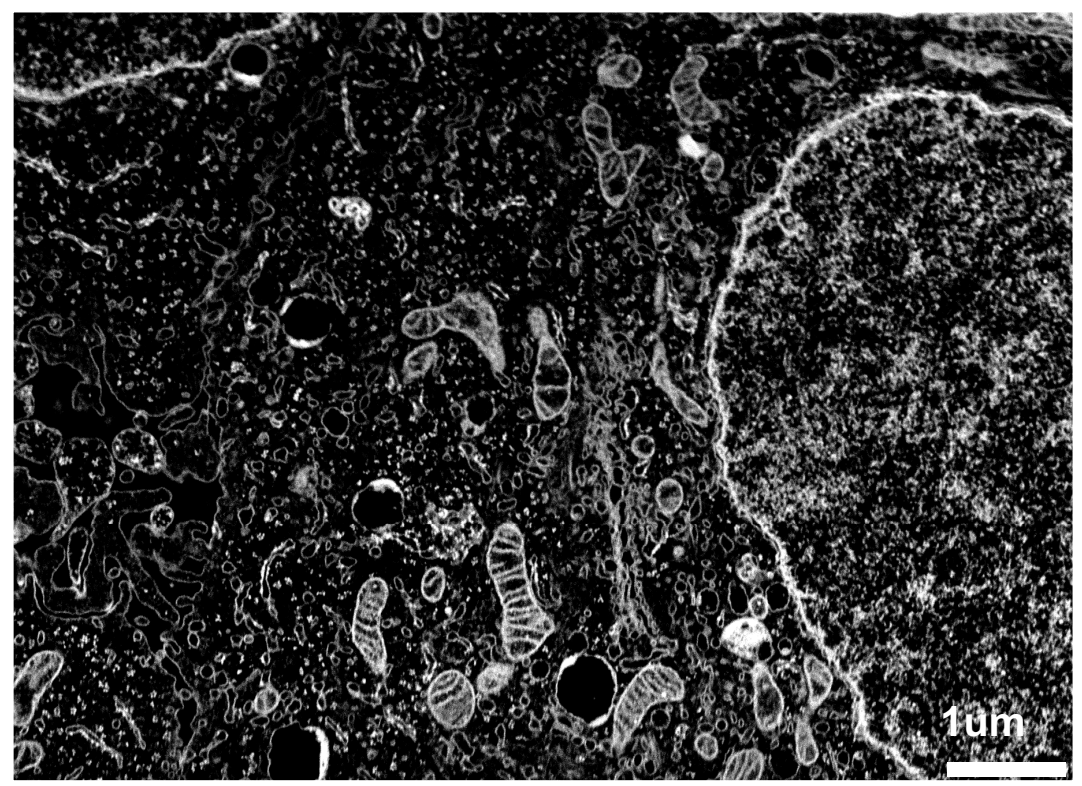

Figure 1 Low-kV back-scattered image of a cancer cell

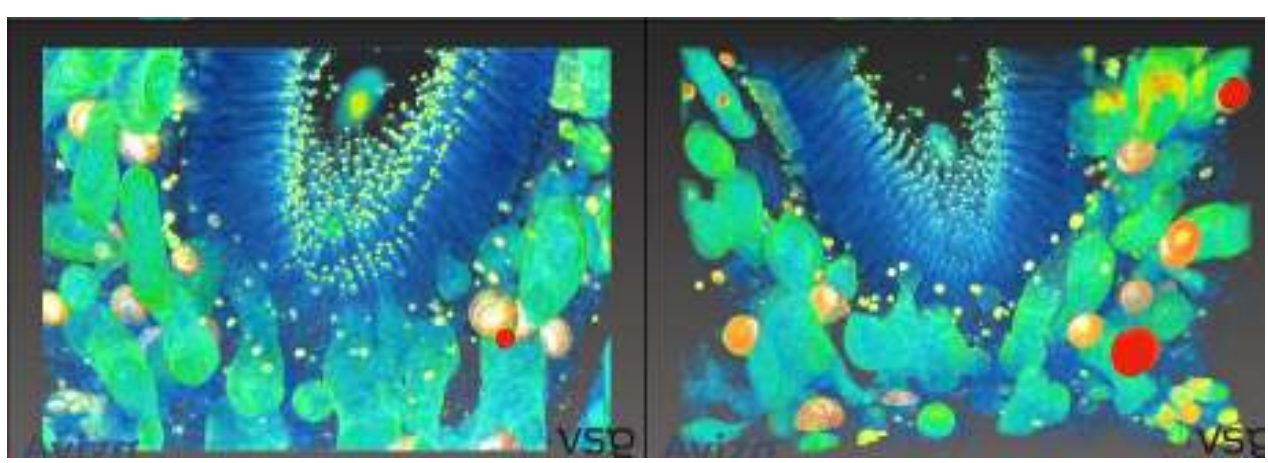

Figure 2 3D Reconstruction of a C. Elegans tissue from FIB nanotomography 